\title{
Photon Kinetics for Laser-Plasma Interactions
}

\author{
L. O. Silva, W. B. Mori, R. Bingham, J. M. Dawson, Thomas M. Antonsen, Jr, Member, IEEE, and P. Mora
}

\begin{abstract}
A photon kinetic formalism is employed to describe the propagation of short laser pulses in tenuous plasmas. The photon kinetic theory contains all of the ingredients necessary to describe the relativistic nonlinear optics of short laser pulses in plasmas, and the shortest time scale is determined by the local evolution of the index of refraction. We use this feature to implement a photon in cell code, in which the typical time step is much bigger than is the laser field time scale. Additional information provided by the photon kinetic framework is illustrated by one-dimensional (1-D) and two-dimensional (2-D) simulations.
\end{abstract}

Index Terms-Intense laser-plasma interactions, numerical simulation, photon kinetics.

\section{INTRODUCTION}

$\mathbf{I}$ $\mathrm{N}$ THE early days of quantum mechanics, the intrinsically wave-like nature of quantum mechanics raised some resistance, and several authors attempted to bridge the gap between the classic picture and the wave formalism. They did this not only to build a more comfortable theory, but also to tackle the problems originating from the statistical nature of quantum mechanics. The most fruitful of these attempts is based on the ideas pioneered by Wigner [1]. Wigner showed that starting from the quantum wave function $\psi$, it is possible to build a phase-space distribution function for the canonical variables position and momentum, whose marginals give the correct description for the physically relevant quantity $\psi \cdot \psi^{*}$, either in configuration or in momentum space. Later, Moyal [2] derived the transport equation for the Wigner distribution function, and Leaf [3] extended the transport equation for Klein-Gordon, and Dirac quantum fields. From the statistical quantum field theory perspective, the Wigner-Moyal formalism is a mature subject [4], [5], with applications ranging from solid-state physics to quantum tomography.

Recent advances in the measurement of ultrashort laser pulses [6], [7] triggered the resurgence of the interest in the phasespace representation of classic fields. Tappert and Besieris [8], [29], [30] first identified the power of the Wigner-Moyal formalism to study classic wave equations, using phase space dis-

Manuscript received December 23, 1999; revised January 20, 2000. This work was supported in part by the National Science Foundation, under Grant AST-9713234 and Grant DMS 9722121, and by the U.S. Department of Energy, under Grant DE-FG03-98DP00211. The work of L. O. Silva was supported by PRAXIS XXI (Portugal). The work of R. Bingham was supported by PPARC (U.K.) and the University of California at Los Angeles.

L. O. Silva, W. B. Mori, and J. M. Dawson are with the Department of Physics and Astronomy, University of California at Los Angeles, Los Angeles, CA 90095 USA (e-mail: silva@physics.ucla.edu).

R. Bingham is with the Rutherford Appleton Laboratory, Chilton, Didcot, OX11 OQX Oxon, U.K.

T. M. Antonsen, Jr. is with the Institute for Plasma Research, University of Maryland, College Park, MD 20742 USA.

P. Mora is with the Centre de Physique Théorique, École Polytechnique, 91128 Palaiseau Cedex, France.

Publisher Item Identifier S 0093-3813(00)07239-8. tribution functions. The field is represented by a Wigner distribution of quasi-particles, and in the limit of short wavelengths, the transport equation is formally equivalent to the Vlasov equation. An analogy between particle dynamics and field dynamics can then be easily established, leading to new, and revealing, physical pictures. A similar formalism has been employed as an extension of the geometrical optics approximation [9] (for electromagnetic wave propagation in random media), or as a generalization of weak turbulence theory for photons [10], [11].

In this paper, we employ the formalism introduced by Tappert to laser propagation in underdense plasmas, and we show that a photon kinetic theory provides all of the ingredients necessary to describe the different stimulated forward scattering instabilities. Based on the collisionless structure of the photon kinetic equation, the natural way to tackle the numerical simulation of photons is a particle-in-cell (PIC) model [12], [13]. In this paper, we describe how such a photon-in-cell code works and present results from a simple one-dimensional (1-D) photon kinetic code that includes dispersion and the relativistic mass correction, but does not include the plasma WAKE response. This code provides illustrative evidence for the formation of envelope solitons as well as informative phase space pictures that characterize this nonlinear structure.

Photon kinetics can be generalized to higher dimensions, and this is illustrated by analogy between the Wigner representation of a Gaussian beam and the distribution function of a particle beam. The quasi-static code WAKE [14] provides the ideal setting to test the principles of photon kinetics in a plasma in higher dimensions, because it is a two-dimensional (2-D) code in which the fast time scale (associated with the high-frequency laser field) is separated for the slow time scale (determined by the plasma dynamics, evolving in the plasma frequency time scale, and the ponderomotive force caused by gradients in the slowly varying laser pulse envelope). We have written a code, called QWAKE, in which the laser field evolution of WAKE (obtained by solving an extended paraxial wave equation for the laser envelope) is replaced by the equivalent photon kinetics. We find excellent agreement between the two approaches. Furthermore, the photon distribution function contains additional information, providing a clear physical picture for laser-plasma interactions. These features will be illustrated with 2-D slab results from QWAKE, the quasi-particle version of WAKE. Finally, the potential and future directions for photon kinetic theory and simulations will also be discussed.

\section{Photon Kinetic Theory}

In order to build a kinetic theory for fields in terms of quasiparticles, it is necessary to address two separate issues. First, a representation of the field in terms of a quasi-particle distribution function in phase space has to be built. Second, it is fun- 
damental to understand how this distribution function is transported. These questions have been answered in a general way in a series of papers by Tappert et al. [8], [29], [30].

The wave equation for the laser field vector potential $\mathbf{A}$ in a plasma can be written as

$$
\left(\partial_{t}^{2}+\Omega^{2}\right) \mathbf{A}=\mathbf{0}
$$

where the positive self-adjoint Hermitian operator $\Omega^{2}$ is defined as

$$
\Omega^{2}=-c^{2} \partial_{x}^{2}+\omega_{p e 0}^{2} \frac{\delta n}{\gamma}
$$

where $\omega_{p e 0}=\left(4 \pi e^{2} n_{0} / m_{e}\right)^{1 / 2}$ is the plasma frequency, $n_{e 0}$ is the background electron density, $\delta n=n_{e} / n_{e 0}$ is the normalized electron density, and $\gamma$ is the relativistic gamma factor of the plasma electrons. It is also assumed that all of the fractional powers of $\Omega^{2}$ are defined, as well as the positive self-adjoint operators. A good discussion about the definition of fractional powers for operators is given in [15]. In general, the fractional powers of operators have to be interpreted as an integral operator.

We define the complex analytic signal as [8], [29]

$$
\psi(\mathbf{x}, t)=\frac{\Omega^{1 / 2}}{\sqrt{8 \pi} c}\left(\mathbf{A}+\frac{i}{\Omega} \partial_{t} \mathbf{A}\right)
$$

The operator $\Omega^{-1}$ is defined such that $\Omega \Omega^{-1}=\overline{\overline{\mathbf{1}}}$. For instance, in vacuum $\Omega=i c \partial_{x}$, and $\Omega^{-1}=-i / c \int d x$. The wave energy density $\epsilon_{\gamma}$, and the wave action density $a_{\gamma}$ associated with $\mathbf{A}$, can then be written as

$$
\begin{aligned}
& \epsilon_{\gamma}=\psi^{t} \cdot \Omega \psi \\
& a_{\gamma}=\psi^{t} \cdot \psi
\end{aligned}
$$

The phase space distribution of the photons $\mathcal{N}$ is the Wigner function of $\psi$

$$
\begin{aligned}
\mathcal{N}(\mathbf{k}, \mathbf{x}, t)= & \int d \mathbf{s} \exp (i \mathbf{k} \cdot \mathbf{s}) \psi\left(\mathbf{x}-\frac{\mathbf{s}}{2}, t\right) \\
& \cdot \psi^{*}\left(\mathbf{x}+\frac{\mathbf{s}}{2}, t\right) .
\end{aligned}
$$

The distribution $\mathcal{N}$ has the good properties of a photon distribution function; i.e., momenta of the distribution function describe observable quantities with the appropriate physical meaning. It is a real-valued function, but not necessarily positive everywhere. The photon number density $n_{\gamma}$, or, equivalently, the classic wave action, which is equivalent to (5), is determined from

$$
\begin{aligned}
n_{\gamma}(\mathbf{x}, t) & =\int \frac{d \mathbf{k}}{(2 \pi)^{3}} \mathcal{N} \\
& =\frac{1}{8 \pi c^{2}}\left(\mathbf{A} \cdot \Omega \mathbf{A}+\partial_{t} \mathbf{A} \cdot \Omega^{-1} \partial_{t} \mathbf{A}\right)
\end{aligned}
$$

The classic wave action is the classic analog of the quantum occupation number, thus, the designation photon number density. It is also possible to define a photon current density $j_{\gamma}$

$$
j_{\gamma}(\mathbf{x}, t)=\int \frac{d \mathbf{k}}{(2 \pi)^{3}} \frac{c \mathbf{k}}{\Omega} \mathcal{N}
$$

and the photon energy density $\epsilon_{\gamma}$ [also determined by (4)]

$$
\begin{aligned}
\epsilon_{\gamma}(\mathbf{x}, t) & =\int \frac{d \mathbf{k}}{(2 \pi)^{3}} \Omega_{W} \mathcal{N} \\
& =\frac{1}{8 \pi c^{2}}\left(\mathbf{A} \cdot \Omega^{2} \mathbf{A}+\left|\partial_{t} \mathbf{A}\right|^{2}\right)
\end{aligned}
$$

where $\Omega_{W}(\mathbf{k}, \mathbf{x}, t)$ is the Weyl transform of the operator $\Omega$ [3], [5], [8]. For a given operator $\hat{A}$, the Weyl transform is defined as [3], [5]

$$
A_{W}(\mathbf{k}, \mathbf{x})=\int d \mathbf{s}\left\langle\mathbf{x}+\frac{1}{2} \mathbf{s}|\hat{A}| \mathbf{x}-\frac{1}{2}\right\rangle \exp (-2 \pi i \mathbf{s} \cdot \mathbf{k})
$$

where $|\mathrm{x}\rangle$ are the eigenkets of the position operator $\hat{x}$; i.e., $\hat{x}|\mathbf{x}\rangle=\mathbf{x}|\mathbf{x}\rangle$. The detailed properties of the Weyl transform are summarized in [5].

The laser field is completely characterized by the distribution $\mathcal{N}$, which can be calculated for different electromagnetic field configurations [16], [17]. Furthermore, there is a one-to-one correspondence between the distribution $\mathcal{N}$, and the analytic signal $\psi$, apart from a constant phase shift $\phi_{0}$. Defining the inverse Fourier transform of $\mathcal{N}$, as

$$
\mathcal{F}\left(\mathrm{x}, \mathrm{x}^{\prime}, t\right)=\int \frac{d \mathbf{k}}{(2 \pi)^{3}} \exp \left(-i \mathrm{k} \cdot \mathrm{x}^{\prime}\right) \mathcal{N}(\mathrm{x}, \mathrm{k}, t)
$$

$\psi$ is determined by

$$
\psi(\mathrm{x}, t)=\frac{\mathcal{F}(\mathrm{x} / 2, \mathrm{x}, t)}{\sqrt{\mathcal{F}(0,0, t)}} e^{i \phi_{0}}
$$

The phase shift $\phi_{0}$ is determined from the initial value conditions, i.e., $e^{i \phi 0}=\psi(\mathbf{x}=0, t=0) / \sqrt{\mathcal{F}(0,0,0)}$, where we have assumed $\mathcal{F}(0,0,0) \neq 0$. Other properties of the Wigner function are compiled in [7].

By providing an alternative picture of the laser field [7], [16], $\mathcal{N}$ already gives additional physical information. However, the full impact of Wigner's formalism can only be achieved if the laser field is replaced by the photon distribution function, and if the wave equation for the laser field is replaced by a transport equation for $\mathcal{N}$. In the underdense limit $\epsilon=\omega_{\text {pe } 0} / \omega \ll 1$, Tappert and Besieris [8], [29], [30] showed that the transport equation for $\mathcal{N}$ is

$$
\frac{\partial \mathcal{N}}{\partial t}+\mathbf{v}_{\gamma} \cdot \frac{\partial \mathcal{N}}{\partial \mathbf{x}}+\mathbf{F}_{\gamma} \cdot \frac{\partial \mathcal{N}}{\partial \mathbf{k}}=O\left(\epsilon^{2}\right)
$$

where

$$
\begin{aligned}
& \mathbf{v}_{\gamma}=\frac{\partial \Omega_{W}}{\partial \mathbf{k}}=\frac{\mathbf{k} c^{2}}{\omega} \\
& \mathbf{F}_{\gamma}=-\frac{\partial \Omega_{W}}{\partial \mathbf{x}}=-\frac{\omega_{p e 0}^{2}}{2 \omega} \nabla \frac{\delta n}{\gamma}
\end{aligned}
$$


We have used the fact that in the underdense limit, $\Omega_{W}$ reduces to

$$
\Omega_{W}=\left(k^{2} c^{2}+\omega_{p e 0}^{2} \frac{\delta n}{\gamma}\right)^{1 / 2}+O\left(\epsilon^{2}\right)
$$

Neglecting the correction terms of order $\epsilon^{n \geq 2}$, (13) states photon number conservation in the phase space $(\mathbf{k}, \mathbf{x})$, and it is formally equivalent to the Vlasov equation. Furthermore, the second (third) term on the LHS of (13) describes dispersion (photon acceleration/transverse focusing), as clearly pictured by (14) and (15). Coupling with the plasma occurs through the photon ponderomotive force, which can be expressed in terms of the distribution $\mathcal{N}$ [17]. The basic ingredients to describe the nonlinear optics of plasmas for short laser pulses at relativistic intensities [18] are all contained in this formalism, and it is possible to rederive all of the relevant temporal growth rates for Raman forward scattering, relativistic self-focusing, envelope self-modulation, and relativistic self-phase modulation for arbitrary laser intensities from the photon kinetic theory [19]. The analogy with particle beams is also evident: using the photon kinetic theory, we are able to describe the laser field evolution as an ensemble of quasi-particles, or a photon beam [20].

\section{Photon Kinetic Simulations}

The Vlasov-like structure of the photon kinetic equation provides the ideal setting to examine photon dynamics under the PIC framework [12], [13]. The basic ideas behind an implementation of a photon kinetic code are straightforward. First of all, it is necessary to specify the initial distribution of photons from the initial laser field, using (3) and (6). The distribution of photons $\mathcal{N}$ is deposited on the grid to calculate quantities relevant to the photon dynamics (such as $\mathbf{A}^{2}$ ), or the plasma dynamics (the photons' ponderomotive potential). If the background plasma is allowed to evolve, the plasma particles are pushed (under the Lorentz force from the self-consistent fields, and the ponderomotive force of the photons), and the self-consistent fields are updated. The quantities necessary to move the photons are now present (namely, the updated index of refraction), and the photons are pushed according to their equations of motion (14), (15). Details of the numerical implementation of a photon-in-cell code will be presented elsewhere [21]. Here, we will concentrate our discussion on some of the qualitative features of the numerical results, and the advantages that photon in cell simulations may provide.

\section{A. One-Dimensional Relativistic Photon Dynamics Without Plasma Response}

The most simple, and nontrivial, scenario that is possible to examine under the photon kinetic formalism is the propagation of an ultraintense laser beam, including the relativistic mass correction nonlinearity, but neglecting the underdense plasma response. This is equivalent to setting $\delta n=1$ in (14)-(16); with $\gamma=\sqrt{1+\mathbf{a}^{2}},\left(\mathbf{a}=e \mathbf{A} / m_{e} c^{2}\right)$, (1) is the nonlinear Klein-Gordon equation, with the potential $V(\mathbf{a})=\left(1+\mathbf{a}^{2}\right)^{1 / 2}$. Furthermore, when $|\mathbf{a}| \ll 1$, and dispersion effects are neglected, the nonlinear Schroedinger equation is obtained. It is then expected that the time evolution of the photon beam first shows relativistic self-phase modulation [18], [22], and then evolves to envelope solitons [23], [24]. Under this scenario, we can demonstrate the key features of photon dynamics in phase space.

We first examine relativistic self-phase modulation (RSPM). Loading a uniform monochromatic photon beam, such that $\mathcal{N}=n_{\gamma 0} \delta\left(\mathbf{k}-\mathbf{k}_{0}\right)$, where $n_{\gamma 0}=\omega_{0} \mathbf{a}^{2}$, with $\omega_{0}=\left(k_{0}^{2} c^{2}+\omega_{p e 0}^{2} / \sqrt{1+\mathbf{a}^{2}}\right)^{1 / 2}$, we expect the maximum growth rate for RSPM to occur for $k=|\mathbf{a}| \omega_{0} / \sqrt{2} c$ [18], [22]. The phase space evolution of the photon distribution is shown in Fig. 1 , for $\mathbf{a}^{2}=0.4$, and $\omega_{0} / \omega_{p}=10$. We first observe the effect of photon acceleration at $\tau=150 / \omega_{p}$, leading to a significant, and symmetric, spread in $\mathbf{k}$ : photon acceleration generates phase-space shear along the $\mathrm{k}$ direction. At this point, dispersion is not playing any role, which means that the fastest growing mode corresponds to the shortest wavenumber allowed in the system. As the wavenumber spread increases, dispersion becomes important (Fig. $1 @ \tau=210 / \omega_{p}$ : photons with higher $k$ move faster: dispersion generates phase-space shear along the $\mathrm{x}$ direction). Fast photons start to overtake the slow photons, leading to photon bunching, which in turn increases the relativistic nonlinearity, thus, closing the feedback loop. However, dispersion is not symmetric in $\mathbf{k}$ [see (14)], thus, leading to detuning between the sidebands, and giving rise to an instability cutoff at $k_{\max }=\omega_{0}|\mathbf{a}| / c$ [18], [22]. Large-scale nonlinear structures are formed, characterized by photon vortices in phase space, as illustrated in Fig. $1 @ \tau=600 / \omega_{p}$, corresponding to six e-foldings for the maximum growth rate. As the photon beam evolves, we observe that the big beam breaks apart in a train of pulses, i.e., solitons, with energy cascading to lower wavenumbers. A typical situation after a long propagation distance is shown in Fig. 2. The usefulness of the photon kinetic description becomes apparent. For long propagation distances, the pulse shape of the individual photon pulses in real space remains almost unaltered, but the phase space evolution is rather complex. The photon bunches form vortices in phase space that clearly shows that dispersion has been compensated by the nonlinearity. The internal phase space structure of one pulse is characteristic of a photon shock. The pulses that have not attained a stationary envelope are still undergoing relativistic self-phase modulation (but now seeded by the pulse envelope gradient). This is clear for the last pulse in Fig. 2. The time evolution also shows that some pulses split in two. This is occurring for pulse 3 , in which the second pulse is "radiating" outward from it.

As expected, the different bunches have different peak $\mathbf{a}^{2}$; this indicates the pulses move with slightly different average velocities (bigger $\mathbf{a}^{2}$ implies faster pulse velocities). Ideally, the long time evolution could result in the formation of a train of identical photon pulses.

\section{B. QWAKE: A Two-Dimensional Quasi-Static Photon Kinetic Code}

WAKE [14] is a 2-D quasi-static PIC code, which evolves the laser field in the plasma dynamical time scale. The obvious advantage of such an approach is the possibility to model ultrashort intense laser pulse propagation in underdense plasmas for 

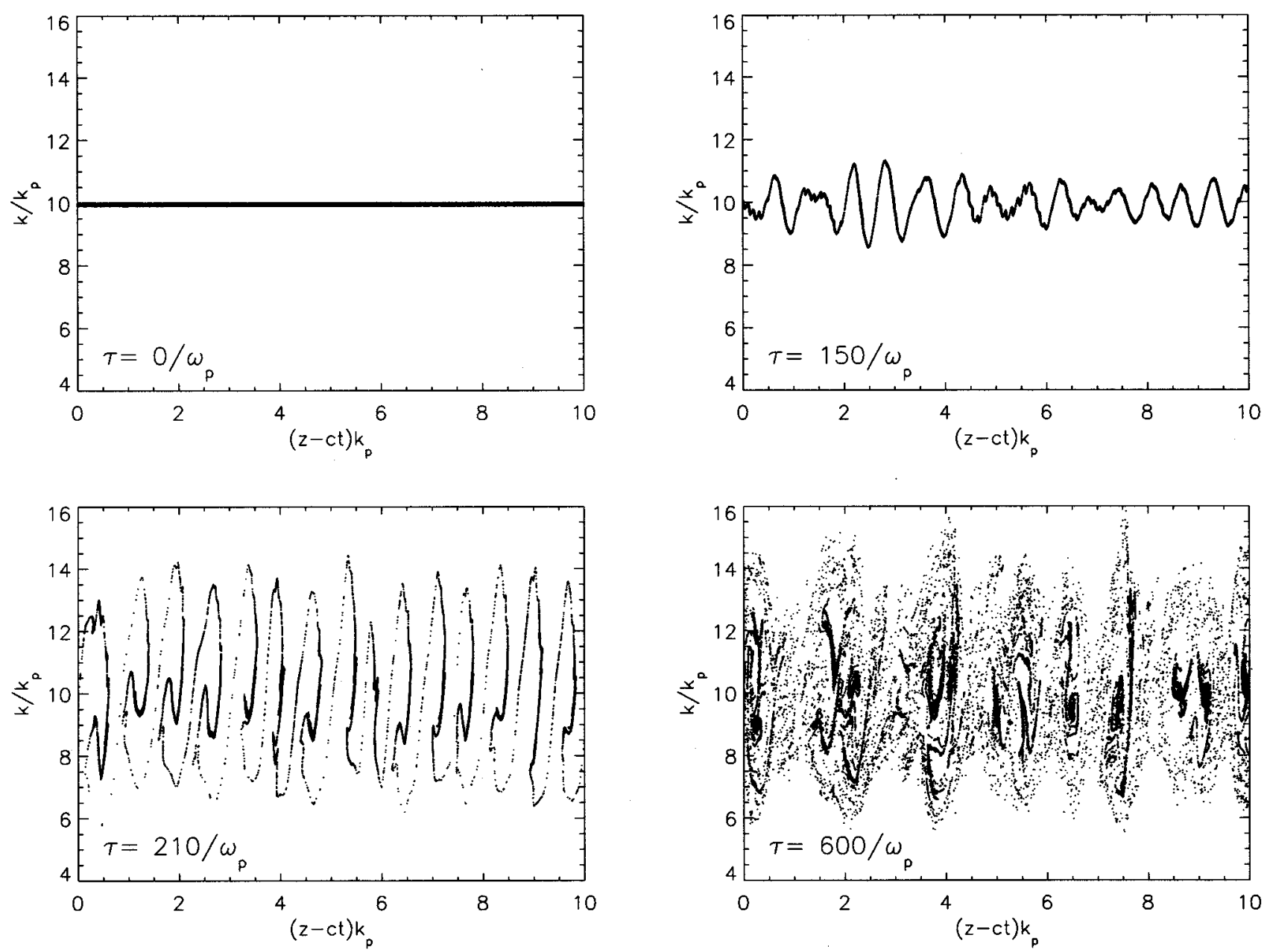

Fig. 1. Phase space evolution of a photon beam, exhibiting relativistic self-phase modulation. Simulation box is three times larger than displayed region, with 300 cells, and $2 \times 10^{4}$ photons, and periodic boundary conditions.

distances comparable to typical laboratory experiments length scales, even with modest computational resources. This is a critical issue to the design of plasma-based accelerators [25], [26]. In WAKE [14], the laser field evolution is decoupled from the plasma self-consistent fields, and because coupling with the plasma is through the laser ponderomotive force, this code provides the ideal framework to test the photon kinetic approach. In WAKE, the laser field envelope is described by an extended paraxial wave equation coupled with the plasma through the changes in the index of refraction of the laser field. In QWAKE, the laser field envelope is a moment of the photon distribution function, and the photon distribution function evolves according to the transport equation (13). The plasma dynamics is treated in the same way in both codes: the plasma particles are under the action of the laser ponderomotive force and the plasma self-consistent fields.

The generalization from 1-D photon kinetics to 2-D slab (or 3-D cartesian) is straightforward, only involving standard numerical techniques related with the generalization of the photon pusher, and the photon density deposition scheme. The key issue here is the determination of the initial photon distribution, which can be calculated numerically from (6). However, for some laser field configurations, it is possible to determine $\mathcal{N}$, and establish a close analogy with particle beams. For a monochromatic plane wave, the photon distribution is trivially calculated, corresponding to a monoenergetic photon beam [16]. It is also possible to calculate the photon distribution for the fundamental mode of a Gaussian beam propagating along $z$ ( $z$ plays here the role of time, and $k_{z}$, the canonical conjugate variable of $z$, plays the role of $\omega$ ), given by $\mathbf{A} \propto \exp \left[i k_{z} \mathbf{x}_{\perp}^{2} / 2 R(z)\right] \exp \left[-\mathbf{x}_{\perp}^{2} / W(z)^{2}\right][20]$

$$
\begin{aligned}
& \mathcal{N}\left(\mathbf{x}_{\perp}, \mathbf{k}_{\perp}, z\right) \\
& \quad \propto \exp -\left[2 \frac{\mathbf{x}_{\perp}^{2}}{W(z)^{2}}\left(1+\alpha^{2}\right)-2 \alpha \mathbf{x}_{\perp} \cdot \mathbf{k}_{\perp}+\mathbf{k}_{\perp}^{2} \frac{W(z)^{2}}{2}\right]
\end{aligned}
$$

where $W(z)=W_{0} \sqrt{1+z^{2} / z_{R}^{2}}$ is the beam waist, $\alpha=k_{z} W(z)^{2} / 2 R(z)$ is the Twiss parameter, with $R(z)=z+z_{R}^{2} / z$ the Gaussian beam radius of curvature, $z_{R}$ is the Rayleigh length, and $W_{0}$ is the minimum spot size. We note that (17) is similar to a Gaussian distribution of free-streaming electrons [20]. $k_{z}$ does not appear explicitly in the photon distribution because for a Gaussian beam with frequency $\omega_{0}, k_{z}$, and $\mathrm{k}_{\perp}$ satisfy the dispersion relation $k_{z}=\omega_{0} / c-\left(\omega_{p e 0}^{2}+\mathbf{k}_{\perp}^{2} c^{2}\right) / 2 \omega_{0} c$. This means that additional 

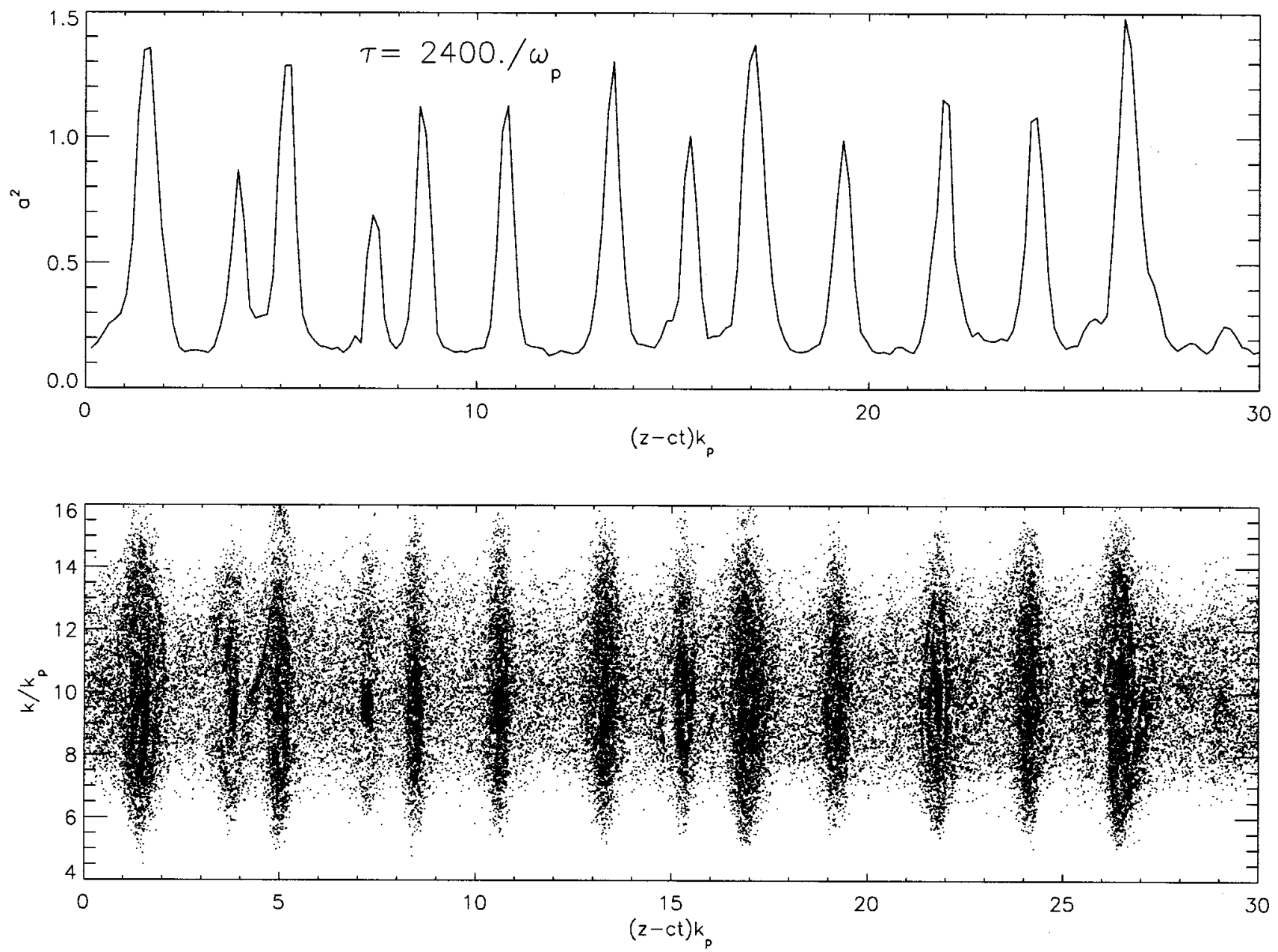

Fig. 2. Quasisatured state of a photon beam: $\mathbf{a}^{2}$ and photons' phase space distribution (peaks referenced in the text from left to right).

dependence on $k_{z}$ is already included in (17) through the connection between $k_{z}$ and $\mathrm{k}_{\perp}$.

We have performed several runs to benchmark the quasi-particle photon kinetic version of WAKE, QWAKE, in 2-D slab geometry. Comparable performances were observed with the two versions, leading to similar quantitative results. In Fig. 3, we present a typical result from QWAKE, portraying the evolution of $\mathbf{a}^{2}$ for a pulse duration $\tau=20 / \omega_{p}$, and a spot size $W_{0}=56.5 c / \omega_{p e 0}$, propagating in a underdense plasma such that $k_{0} / k_{p e 0}=11$, with $\mathbf{a}_{\max }^{2}(\tau=0)=0.25$. The result from WAKE for the same parameters (but with a finer transverse grid) is presented in Fig. 3(c). The results from QWAKE and WAKE show good qualitative agreement. In particular, the longitudinal profile along the laser pulse propagation axis comparing QWAKE and WAKE (Fig. 4) shows equivalent qualitative features, but small quantitative differences. However, we observe that the QWAKE results are almost independent of the transverse resolution, and the WAKE results were obtained for a very high transverse resolution run. Furthermore, QWAKE is not yet optimized for the photon dynamics, and the five-passage binomial smoother [13] along the $z$-direction prevents the observation of the finer details shown by the WAKE run. Detailed comparisons between the two approaches will be presented in a future publication [21].
For the conditions in Fig. 4, relativistic self-focusing leads to intensity enhancement and eventually to pulse splitting and pulse compression of the front part of the laser pulse [27]. This physical interpretation becomes more clear if additional diagnostics over the photon distribution $\mathcal{N}$ are calculated. It is possible to define averages over $\mathcal{N}$, for a given observable quantity $f$, as

$$
\langle f\rangle(\mathbf{x}, t)=\frac{\int d \mathbf{k} f \mathcal{N}}{\int d \mathbf{k} \mathcal{N}}
$$

in configuration space, whereas in momentum space

$$
\langle f\rangle(\mathrm{k}, t)=\frac{\int d \mathrm{x} f \mathcal{N}}{\int d \mathbf{x} \mathcal{N}} .
$$

Physical quantities with significant relevance are, for instance, the local average of the photons' frequency $\langle\omega\rangle$, and the local average of the photons' perpendicular momentum $\left\langle k_{\perp}\right\rangle$. The first quantity gives a measure of the laser field chirp and the effect of photon acceleration. The second quantity 

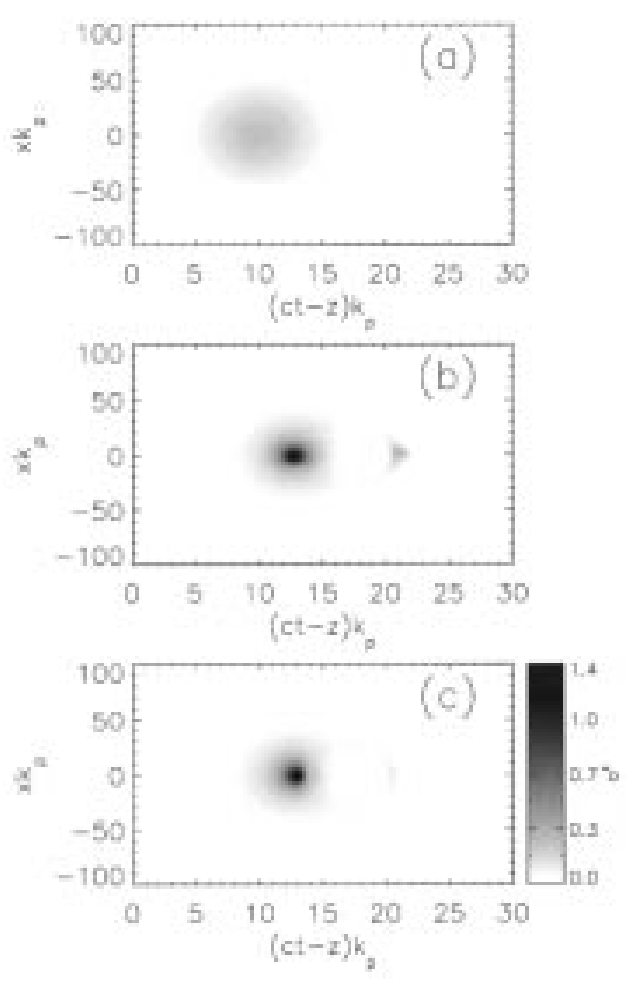

Fig. 3. $\mathbf{a}^{2}$ from QWAKE and WAKE: (a) Initial $\mathbf{a}^{2}$; (b) $\mathbf{a}^{2}$ obtained from propagating the photon distribution function after 0.04 Rayleigh lengths; (c) same conditions as in (b), but using WAKE i.e., solving directly for the laser field wave equation. For QWAKE, the simulation box is 100 cells wide (and 200 cells long), with $2 \times 10^{5}$ photons, and a moving window, whereas for WAKE the simulation box is 300 cells wide (and 200 cells long).

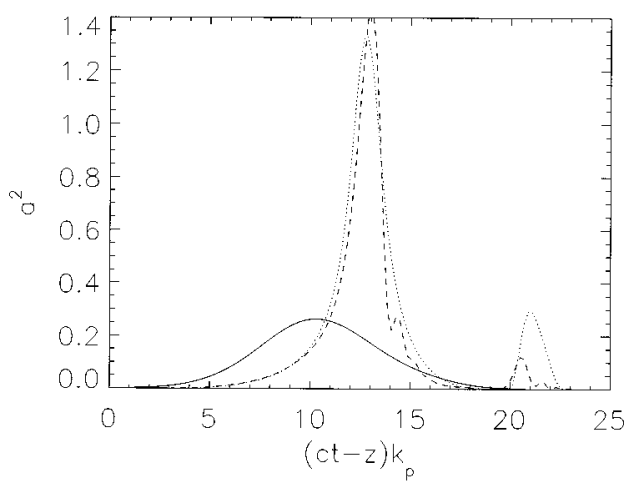

Fig. 4. $\mathbf{a}^{2}$ along the laser propagation axis $(c t-z) k_{p}$ for the conditions of Fig. 3(a)—solid line, Fig. 3(c)—short dashed line, and Fig. 3(c)—long dashed line.

indicates which regions of the laser field are focusing or defocusing, depending on if $\left\langle k_{\perp}\right\rangle$ is pointing inward, to the laser beam propagation axis, or outward. This is the relevant quantity to examine relativistic self-focusing, as illustrated in Fig. 5. We observe that the enhanced front part of the pulse is self-focusing, whereas pulse splitting is occurring in the defocusing (or diffracting) region, and pulse splitting should occur in this region. These zones are correlated with the appropriate region of the index of refraction profile leading to focusing/defocusing.

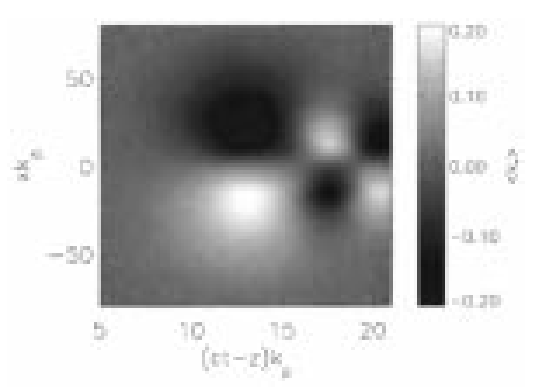

Fig. 5. Local $k_{x}$ determined from the laser field photon distribution for the conditions of Fig. 3(b).

\section{SUMMARY AND CONCLUSION}

Photon kinetic theory provides a novel framework for the study of intense electromagnetic fields interacting with underdense plasmas, in particular, when forward scattering instabilities dominate the plasma dynamics. The most powerful features are its simplicity, the strong connection with particle beam dynamics, and the natural way in which broadband and angular spread effects can be included in the formalism: under this new light, intense laser-plasma interactions are a combination of very well-defined physical processes [18]. The perfect setting for photon kinetic theory is high brightness, broadband, radiation-driven plasma instabilities, relevant for inertial confinement fusion and astrophysics [19].

The strong analogy of photon dynamics with particle dynamics leads to an obvious path toward the numerical realization of a photon kinetic code. Furthermore, the generalization to higher dimensions is easy, only relying on standard techniques. Thus, it might circumvent numerical difficulties associated with the solution of the wave equation in three dimensions, in reduced PIC codes [28].

With a 1-D photon kinetic code, we have analyzed some of the qualitative features of RSFM and the formation of envelope solitons. The photon phase space provides signatures for these nonlinear structures. By modifying WAKE to evolve a photon distribution coupled with the plasma, we were able to benchmark photon kinetics against a well-tested code. Excellent qualitative agreement was found between the two versions of the code, and the similar quantitative results show that photon kinetics is a promising representation for short laser pulse propagation in underdense plasmas. The additional diagnostics associated with photon kinetics established clear physical pictures for previous analytical, and numerical results [18], [27]. We have demonstrated that photon kinetic PIC codes include all of the fundamental physics, open the way to new photon diagnostics, and are computationally competitive with other reduced algorithms for short-pulse laser-plasma interactions.

\section{ACKNOWLEDGMENT}

The authors would like to thank B. Afeyan for useful discussions.

\section{REFERENCES}

[1] E. Wigner, "On the quantum correction for thermodynamic equilibrium," Phys. Rev., vol. 40, pp. 749-759, 1932. 
[2] J. E. Moyal, "Quantum mechanics as a statistical theory," in Proc. Cambridge Phil. Soc., vol. 45, 1949, p. 99.

[3] B. Leaf, "Weyl transformation and the classical limit of quantum mechanics," J. Math. Phys., vol. 9, p. 65, 1968.

[4] R. P. Feynman, Statistical Mechanics: A Set of Lectures. Redwood City, CA: Addison-Wesley, 1972

[5] R. L. Liboff, Kinetic Theory. New York: Wiley, 1998.

[6] D. J. Kane and R. Trebino, "Single-shot measurement of the intensity and phase of an arbitrary ultrashort pulse by using frequency-resolved optical gating," Opt. Lett., vol. 18, pp. 823-825, 1993.

[7] J. Paye, "The chronocyclic representation of ultrashort light pulses," IEEE J. Quantum Electron., vol. 28, pp. 2262-2273, 1992.

[8] F. D. Tappert, "Derivation of the collisionless wave kinetic equation," SIAM Rev. (Chronicle), vol. 13, p. 281, 1971.

[9] S. W. McDonald, "Phase-space representation of wave equations with applications to the eikonal approximation for short-wavelength waves," Phys. Rep., vol. 159, p. 337, 1988

[10] R. Bingham, J. T. Mendonça, and J. M. Dawson, "Photon Landau damping," Phys. Rev. Lett., vol. 78, pp. 247-249, 1997.

[11] N. L. Tsintsadze and J. T. Mendonça, "Kinetic theory of photons in a plasma," Phys. Plasmas, vol. 5, pp. 3609-3614, 1998.

[12] J. M. Dawson, "Particle simulation of plasmas," Rev. Mod. Phys., vol. 55, p. 403,1983

[13] C. K. Birdsall and A. B. Langdon, Plasma Physics Via Computer Simulation. New York: McGraw-Hill, 1985.

[14] P. Mora and T. M. Antonsen, Jr., "Kinetic modeling of intense, short laser pulses propagating in tenuous plasmas," Phys. Plasmas, vol. 1, pp. 217-229, 1997

[15] L. Fishman and J. J. McCoy, "Derivation and application of extended parabolic wave theories. I. The factorized Helmholtz equation," J. Math. Phys., vol. 25, pp. 285-296, 1984.

[16] L. O. Silva and J. T. Mendonça, "Kinetic theory of photon acceleration: Time-dependent spectral evolution of ultrashort laser pulses," Phys. Rev. $E$, vol. 57, pp. 3423-3431, 1998.

[17] L. O. Silva, R. Bingham, J. M. Dawson, and W. B. Mori, "Ponderomotive force of quasiparticles in a plasma," Phys. Rev. E, vol. 59, pp. 2273-2280, 1999

[18] W. B. Mori, "The physics of the nonlinear optics of plasmas at relativistic intensities for short-pulse lasers," IEEE J. Quantum Electron., vol. 33, pp. 1942-1953, 1997.

[19] L. O. Silva et al., Raman forward scattering by high brightness broadband radiation sources. to be published.

[20] - Quasi-particle representation of photon beams. to be published.

[21] — A photon in cell code for intense radiation-plasma interactions, submitted for publication.

[22] C. Max, J. Arons, and A. B. Langdon, "Self-modulation and self-focusing of electromagnetic waves in plasmas," Phys. Rev. Lett., vol. 33, pp. 209-212, 1974

[23] V. I. Karpman and E. M. Kruskal, "Modulated waves in nonlinear dispersive media," Sov. Phys. JETP, vol. 28, p. 277, 1969.

[24] P. K. Kaw, A. Sen, and T. Katsouleas, "Nonlinear 1D laser pulse solitons in a plasma," Phys. Rev. Lett., vol. 68, pp. 3172-3175, 1992.

[25] T. Tajima and J. M. Dawson, "Laser electron accelerator," Phys. Rev. Lett., vol. 43, pp. 267-270, 1979.

[26] E. Esarey, P. Sprangle, J. Krall, and A. Ting, "Overview of plasma-based accelerator concepts," IEEE Trans. Plasma Sci., vol. 24, pp. 252-288, 1996.

[27] C. Ren et al., Compressing and focusing a short laser pulse by a thin plasma lens. to be published.
[28] D. Gordon, W. B. Mori, and T. M. Antonsen, "A ponderomotive guiding center particle-in-cell code for efficient modeling of laser-plasma interoctions," IEEE Trans. Plasma Sci., vol. 4, pp. 1134-1142, Aug. 2000.

[29] I. M. Besieris and F. D. Tappert, "Propagation of frequency modulated pulses in a randomly stratified plasma," J. Math. Phys., vol. 14, pp. 704-707, 1973.

[30] _ "Stochastic wave-kinetic theory in the Liouville approximation," J. Math. Phys., vol. 17, pp. 734-743, 1976.

[31] F. S. Tsung et al., Generation of single cycle pulses by photon deceleration. to be published.

L. O. Silva, photograph and biography not available at the time of publication.

W. B. Mori, photograph and biography not available at the time of publication.

R. Bingham, photograph and biography not available at the time of publication.

J. M. Dawson, photograph and biography not available at the time of publication.

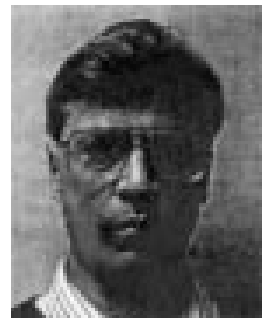

Thomas M. Antonsen, Jr. (M'87) was born in Hackensack, NJ, in 1950. He received the B.S. degree in electrical engineering in 1973, and the M.S. and Ph.D. degrees in 1976 and 1977, all from Cornell University, Ithaca, NY.

He was a National Research Council Post Doctoral Fellow at the Naval Research Laboratory in 1976-1977, and a Research Scientist in the Research Laboratory of Electronics at MIT from 1977 to 1980. In 1980, he moved to the University of Maryland where he joined the faculty of the Departments of Electrical Engineering and Physics in 1984. He is currently a Professor of physics and electrical engineering. He has held visiting appointments at the Institute for Theoretical Physics (UCSB), the Ecole Polytechnique Federale de Lausanne, Switzerland, and the Institute de Physique Theorique, Ecole Polytechnique, Palaiseau, France. His research interests include the theory of magnetically confined plasmas, the theory and design of high power sources of coherent radiation, nonlinear dynamics in fluids, and the theory of the interaction of intense laser pulses and plasmas. He is the author and coauthor of more than 180 journal articles and coauthor of the book Principles of Free-electron Lasers. He has served on the editorial board of Physical Review Letters, The Physics of Fluids, and Comments on Plasma Physics.

Prof. Antonsen was selected as a Fellow of the Division of Plasma Physics of the American Physical Society in 1986.

P. Mora, photograph and biography not available at the time of publication. 